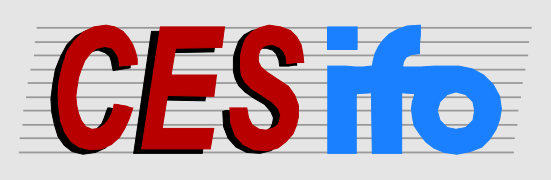

\title{
Working
}

Papers

www.cesifo.org/wp

\section{Green Paradox and Directed Technical Change: The Effect of Subsidies to Clean R\&D}

\author{
Julien Daubanes \\ André Grimaud \\ Luc Rougé
}

\author{
CESIFO WORKING PAPER NO. 4334 \\ CATEGORY 9: RESOURCE AND ENVIRONMENT ECONOMICS \\ JULY 2013
}

An electronic version of the paper may be downloaded

- from the SSRN website:

- from the RePEc website:

- from the CESifo website:

www.SSRN.com

www.RePEc.org

www.CESifo-group.org/wp

\section{CESifo}




\title{
Green Paradox and Directed Technical Change: The Effect of Subsidies to Clean R\&D
}

\begin{abstract}
We borrow standard assumptions from the non-renewable-resource-taxation and from the directed-technical-change literatures, to take a full account of the incentives to perform $R \& D$ activities in a dirty-resource sector and in a clean-resource-substitute sector. We show that a gradual rise in the subsidies to clean $R \& D$ activities causes a less rapid resource extraction, because it enhances the long-run resource productivity. Our result contradicts the greenparadox conjecture that technical improvements in resource substitutes accelerate resource extraction. Sector-specific innovation activities are tantamount to competing economic projects; general equilibrium with several $R \& D$ sectors implies no-arbitrage conditions that give rise to not-so-intuitive results.
\end{abstract}

JEL-Code: Q320, O320, O410.

Keywords: non-renewable resources, directed technical change, green paradox, environmental policy, R\&D subsidies.

\author{
Julien Daubanes \\ Center of Economic Research (CER-ETH) \\ Swiss Federal Institute of Technology \\ Switzerland / Zurich \\ jdaubanes@ethz.ch
}

\author{
André Grimaud \\ Toulouse School of Economics (IDEI \& \\ LERNA) / Toulouse Business School \\ France / Toulouse \\ andre.grimaud@tse-fr.eu
}

\author{
Luc Rougé \\ University of Toulouse \\ Toulouse Business School \\ France / Toulouse \\ l.rouge@esc-toulouse.fr
}

July 2013

Particular thanks go to Sjak Smulders for his very helpful suggestions on an earlier version. We also thank Louis-Gaëtan Giraudet and participants at various seminars and conferences: Annual Congress of the French Economic Association 2011; Rencontres de l'Environnement 2011 at Paris School of Economics; Toulouse Business School 2012; SURED Conference 2012; Annual Congress of the EAERE 2013 at Toulouse School of Economics. 


\section{Introduction}

The so-called "green paradox" (Sinn, 2008) phenomenon refers to the fact that anticipated policies aimed to reduce the demand for an exhaustible resource result in this resource being exploited more rapidly. It is well known that when such resources are polluting - as are fossil fuels - free markets already tend to consume them too rapidly (Withagen, 1994). Hence, policies which entail a green paradox phenomenon further deteriorate the environment and are thus obviously sub-optimal. ${ }^{1}$ Hans-Werner Sinn coined the expression, meaning that "good intentions do not always breed good deeds" (p. 380).

Such phenomena may arise from several types of demand-reducing policies. In his formal analysis, Sinn (2008) has focused on taxation policies applied to a non-renewable resource when those policies leave the total amount to be ultimately extracted unaffected. In such Hotellian contexts, as he shows, tax instruments that are sufficiently rising over time whether they are applied on cash flows or directly on resource quantities - induce a more rapid extraction. ${ }^{2}$ Thus, an anticipated gradual introduction of environmental policies, or the anticipation that environmental policies will be implemented from some future dates on, might result in an undesired faster extraction.

Sinn (2008) anticipated that the result should carry over to the case of any demandreducing policies; in particular, to subsidies to resource substitutes and to technical improve-

\footnotetext{
${ }^{1}$ An exact evaluation of policies' environmental impacts requires an explicit assessment of their effects on pollution and on social welfare (e.g. Hoel, 2010; Gerlagh, 2011; van der Ploeg and Withagen, 2012). Following Sinn (2008), Grafton, Kompas and Long (2012) and others, a meaningful simplification consists in assessing whether policies enhance or reduce the speed at which the resource is extracted. Environmental policies which contribute to solving the environmental problem slow down the extraction of polluting resources; vice versa policies inducing a more rapid extraction are detrimental to the environment. This simple criterion has been considered to give a good intuition on whether policies are environmentally successful or not, contributing to the popularity of the green paradox problem as initially formulated by Sinn (2008).

${ }^{2}$ The reasoning goes as follows. A constant tax rate applied to cash flows is neutral because it amounts to a conventional profit tax on the total-discounted-profit objective. Absent any extraction cost, a constantpresent-value levy on the resource is formally identical to a constant cash-flow tax. Even with non-zero extraction costs, there exists a continuum of neutral tax paths (Dasgupta, Heal and Stiglitz, 1981). All those extraction taxes are neutral for the same reason that they affect the equilibrium price in such a way that the equilibrium Hotelling rule remains satisfied without any further readjustments of quantities; in particular, they leave the producers' profit-maximizing extraction unchanged. Tax trajectories that are rising more rapidly than those neutral ones cause a more rapid extraction (see also the comprehensive analysis of Gaudet and Lasserre, 1990).
} 
ments in the production of those substitutes. Most of following contributions have dealt with subsidies to resources' clean substitutes while extending the traditional partial-equilibrium resource-depletion setting in various respects (e.g. Gerlagh, 2011; Grafton, Kompas and Long, 2012; van der Ploeg and Withagen, 2012; and others). ${ }^{3}$

There are three basic ways by which the demand for a commodity can be affected. The first one is to directly modify its price through taxation policies directly applied on its flows, in the spirit of Sinn (2008). Quite relatedly, the second one is to affect the price of its substitutes, in the spirit of Gerlagh (2011), Grafton et al. (2012) and van der Ploeg and Withagen (2012). Those two ways consist in modifying the price arguments of the demand function; using partial-equilibrium settings where the technology is given, they have been extensively analyzed by the above studies.

The third way to affect the demand for a commodity is the object of this paper: it consists in modifying the demand function itself instead of its price arguments. Macroeconomic demand drivers are arguably as strong as price incentives in the determination of the global demand for fossil fuels. Demand depends on the currently available technology; in a long-run perspective, the technology results from prior research and development (R\&D) investments. The long-run dynamic mechanisms by which private research efforts respond to economic incentives have been highlighted by the economic growth literature. As this literature shows, such economic incentives do not affect all sectors in the same fashion (Acemoglu, 2002): research investments are directed to specific sectors; understanding the intricate process by which a particular sector is affected by endogenous $R \& D$ requires to assume that innovations are sector specific.

Acemoglu's concept of directed technical change has received a particular attention in resource economics. Indeed, directed-technical-change models disentangle the specific factors favoring the production of clean substitutes from those enhancing the productivity of dirty

\footnotetext{
${ }^{3}$ Grafton et al. (2012) introduced decreasing returns to scale in a substitute production, arising from landsupply limitations. Gerlagh (2011) and van der Ploeg and Withagen (2012) departed from the Hotelling assumption of resource homogeneity and examined the induced dynamics of pollution.
} 
resources. Then, by refining the patterns of substitutability and complementarity between sectors, it permits to more precisely describe the role of $R \& D$ in the switch from the use of depletable resources to the use of producible (gross) substitutes. Major applications include, among others, André and Smulders (2004), Hart (2004), Grimaud and Rougé (2008), Di Maria and Valente (2008), Pittel and Bretschger (2010) and Gans (2012). A recent and particularly noteworthy contribution on the issue is Acemoglu, Aghion, Bursztyn and Hemous' (2012) paper.

Subsidies to research investments are generally advocated by the economic growth literature $^{4}$, yet irrespective of whether these investments aim to favor clean or dirty sectors. Subsidies to environmentally friendly research activities - enhancing productivity in nonpolluting substitute sectors - affect the demand of polluting-resource inputs only indirectly; therefore, absent any constraints on the implementation of optimal environmental policies, they must not have any Pigovian dimension. In the presence of policy implementation difficulties however (perhaps related to green paradox phenomena), they may contribute to alleviate the environmental problem arising from polluting-resource use. On this ground, they can be advocated as not-too-bad substitutes to direct environmental policies (e.g. Grimaud, Lafforgue and Magné, 2011). In general, as Smulders and Di Maria (2012) recently pointed out, induced technical change interacts with pollution-generating inputs' demand in a very intricate way. ${ }^{5}$

Intellectual property rights confer sector-specific innovation activities the dimension of competing economic projects. The equilibrium allocation of efforts to polluting-resource-

\footnotetext{
${ }^{4}$ By providing incentives to increase otherwise sub-optimal R\&D investments, they alleviate standard endogenous-growth distortions.

${ }^{5}$ As they show, the interaction between environmental policies and technological changes they induce leads to counter-intuitive effects even in models where there is a single aggregated R\&D sector. Their analysis mainly focuses on the effects of environmental policies, taking into account endogenous technological adjustments. In contrast, we focus on policies directly supporting a specific R\&D sector. In the standard two-sector CES-technology framework of the directed-technical-change literature, exogenous technical improvements in the clean sector can be shown to satisfy Smulders and Di Maria's definition of "brown" technologies regardless of sectors' substitutability/complementarity. In our intertemporal Hotelling model, extraction patterns are not only governed by the absolute effect of technological change on resource demand, but further depend on these effects at all dates relative to each other.
} 
improving and to clean-substitute-improving R\&D sectors is determined by no-arbitrage conditions involving the inclusive-of-subsidies returns to investments in both sectors. Besides their general economic interest, such interior equilibria have an obvious empirical relevance: as a matter of fact, specific R\&D activities currently take place simultaneously in resource and non-resource sectors (e.g. Aghion, Dechezlepretre, Hemous, Martin and Van Reenen, 2011). ${ }^{6}$ Yet, they are rarely examined by the directed-technical-change literature (e.g. Acemoglu et al., 2012; Gans, 2012). ${ }^{7}$ We show that when public policies sufficiently support clean-substitute-improving $\mathrm{R} \& \mathrm{D}$, interior equilibria arise in finite time as the economy's long-run path. We focus on policy-induced changes on these long-run interior equilibria.

In this context, we find that the effects of relative subsidies to research efforts are not so intuitive. The equilibrium no-arbitrage requirement implies that any support to one specific R\&D sector entails necessary compensations to the other sectors. As a result, depending on whether production sectors are substitutable or complementary, supporting the clean-substitute R\&D sector affects positively or negatively the relative contribution of the clean-substitute production sector. However, the overall effect of R\&D-support policies on resource demand is irrespective of sectors' degree of substitutability/complementarity. As we show, a gradual, more-and-more stringent support to R\&D activities aimed to improve productivity in clean-substitute sectors always induces, among other effects, a less rapid resource extraction. Our result sharply contrasts with the commonly-made conjecture that technical improvements in the production of resource substitutes are tantamount to other policies aimed to reduce the demand for the resource.

Taking a full account of incentives to perform R\&D requires to consider the endogenous process by which the productivity of both the resource and its substitutes is determined by specific R\&D sectors. Our analysis suggests that the endogeneity of technical change is

\footnotetext{
${ }^{6}$ Aghion et al. (2011) specifically study the patents granted over the same period that are related to the traditional motor-fuel automotive technologies and to their substitute (hybrid, electric) technologies.

${ }^{7}$ For instance, Acemoglu et al. (2012) focus on corner equilibria where innovation activities only take place in one R\&D sector; Gans (2012) considers innovation activities in all sectors, yet in isolation of each other.
} 
crucial to study the effects of supporting the development of resource and resource-substitute sectors. To set up our model, we borrow very standard assumptions from the recent literature on directed technical change and the environment; our framework is largely inspired from Acemoglu et al.'s (2012) analysis.

We adopt their setup and modify it so as to examine the effects of specific R\&D-support policies on non-renewable-resource extraction patterns in a single model involving the minimal ingredients determining those effects: two production sectors combine to produce a unique final good; each of them is associated with a specific R\&D sector; one of them (dirty) consumes flows of a non-renewable resource; the other one (clean) relies on flows of a renewable source of energy.

Unlike Acemoglu et al., the time dimension is continuous, which requires some minor adjustments which basically amount to a redefinition of the time scale. For simplicity, we assume away the allocation of unskilled labor to the two production sectors. The allocation of economic resources to these sectors is completely summarized by that of intermediate goods. Nevertheless, the allocation of research (labor) efforts to the two R\&D sectors retains its central role identified by the directed-technical-change literature.

In general-equilibrium models of endogenous growth that integrate an exhaustible-resource sector, it is standard to assume that extraction costs are negligible. ${ }^{8}$ With such a common approximation, traditional growth models still deliver good intuitions while preserving the regularity properties which are required in a long-run-growth perspective. In general, environmental policies affect the ultimately extracted resource quantity, either because some reserve units become uneconomic (e.g. Hoel, 2010; Gerlagh, 2011; van der Ploeg and Withagen, 2012) or because of lower exploration and development efforts (e.g. Daubanes and Lasserre, 2012). ${ }^{9}$ For its purpose is to emphasize the role of R\&D processes in a long-rungrowth perspective, the present paper gives priority to the study of policies' effects on the

\footnotetext{
${ }^{8}$ A notable exception is André and Smulders (2004). Still, their specification does not make the ultimately extracted quantity responsive to policies since the entire stock is always exhausted over the horizon.

${ }^{9}$ In either case, this amounts to take the heterogenity of deposits into account.
} 
speed of resource exploitation, at the expense of those on how much is ultimately depleted.

As the above contributions show, a reduction of total extraction can easily be achieved with conventional environmental policies applied to the resource or to its substitutes directly.

The analysis goes as follows. Section 2 exposes the setup. Section 3 analyzes the function by which resource demand is determined; it identifies its main macroeconomic driver as the relative importance of the resource and non-resource sectors. Section 4 focuses on the effects of increased relative subsidies to clean R\&D activities on the economy's structure. Section 5 combines the results of Sections 3 and 4 and integrates the intertemporal dimension of resource extraction and of economic growth to deliver the main message of the paper. Section 6 concludes.

\section{The Model}

At each date $t$ of the continuous set $[0,+\infty)$, the competitive final sector produces a quantity $Y(t)$ of final good using a "clean" input $Y_{c}(t)$ and a "dirty" input $Y_{d}(t)$, according to the CES aggregate production function

$$
Y(t)=\left[Y_{c}(t)^{(\varepsilon-1) / \varepsilon}+Y_{d}(t)^{(\varepsilon-1) / \varepsilon}\right]^{\varepsilon /(\varepsilon-1)}
$$

where $\varepsilon \in(0,+\infty)$ is the elasticity of substitution between the clean and dirty production sectors.

The clean sector competitively produces the output $Y_{c}(t)$ from a flow $Q(t)$ of renewable energy supposed to be non polluting (e.g. solar, wind energies). For simplicity, we assume this flow $Q(t)$ to be constant, equal to $Q$, as if it was produced from a constant flow of the archetypical renewable labor energy. ${ }^{10}$

\footnotetext{
${ }^{10}$ For instance, $Q(t)$ is produced from the labor quantity $L_{Q}(t)$ only, according to the constant-return-toscale production function $Q(t)=\beta L_{Q}(t)$, with $L_{Q}(t)=L_{Q}$. Also for simplicity, the use of the resource input $R(t)$ in the other (dirty) sector will not require any labor energy. Had we assumed a flow of homogeneous labor to be allocated to the clean and dirty sectors, as in Acemoglu et al. (2012), our results would not have changed in any fundamental manner. Indeed, what ultimately matters for the equilibrium allocation to exhibit a trade-off between the two sectors is that some inputs - at least one - are used in both of them. As will be clear later on, these inputs are the intermediate ones.
} 
The dirty sector's output $Y_{d}(t)$ is competitively produced from a flow $R(t)$ of nonrenewable resource supposed to be polluting (e.g. fossil fuels). ${ }^{11}$ This flow is costlessly extracted from a fixed stock $S_{0}$ of Hotelling reserves:

$$
\dot{S}(t)=-R(t)
$$

where $S(t)$ is the remaining stock of reserves to be exploited at date $t \geq 0$ and where, as in the rest of the paper, a dot on top of a variable means that this variable is differentiated with respect to time.

Precisely, the clean and dirty sectors respectively produce $Y_{c}(t)$ and $Y_{d}(t)$ according to the production functions

$$
\begin{aligned}
& Y_{c}(t)=Q^{1-\alpha} \int_{0}^{1} A_{c i}(t)^{1-\alpha} x_{c i}(t)^{\alpha} d i, \\
& Y_{d}(t)=R(t)^{1-\alpha} \int_{0}^{1} A_{d i}(t)^{1-\alpha} x_{d i}(t)^{\alpha} d i,
\end{aligned}
$$

with $0<\alpha<1$. For each sector $j=c, d$ there is a continuum of sector-specific intermediate goods indexed by $i \in[0,1]: x_{j i}(t)$ denotes the quantity of the intermediate good $i$ used in sector $j .{ }^{12}$ Moreover, $A_{j i}(t)$ denotes the contemporaneous quality level of this intermediate good.

Technical change is directed in the sense of Acemoglu (2002): there are two R\&D sectors, one "clean" and one "dirty", respectively associated with the clean and the dirty production sectors. These two R\&D sectors are Schumpeterian as in Aghion and Howitt (1992): they improve the quality level $A_{j i}(t)$ of intermediate goods specific to their respective sectors.

In each R\&D sector $j=c, d$ a number $L_{j i}(t)$ of atomistic scientists are dedicated to improving the quality level $A_{j i}(t)$ of the intermediate good $i \in[0,1]$. Each scientist in the R\&D sector $j$ has an instantaneous time-invariant and sector-specific probability $\eta_{j} \in(0,1)$

\footnotetext{
${ }^{11}$ The analysis focuses on the policy-induced changes in the extraction pattern, in equilibrium outcomes where pollution is not internalized and in absence of direct externality-corrective policies. Thus, the polluting character of the resource need not be made explicit. All along this character will remain implicit.

${ }^{12}$ For example, an intermediate good in the dirty sector $j=d$ may be a type of conventional gas engine; an intermediate good in the clean sector $j=c$ may be a type of solar panel.
} 
of being the successful innovator. In case such a success occurs in sector $j$ on the part of one of the $L_{j i}(t)$ scientists, the quality level $A_{j i}(t)$ rises by $\gamma A_{j i}(t)$ with $\gamma>0$, which means that the new version of the associated intermediate good is more productive; otherwise, that is absent any such success, $A_{j i}(t)$ remains unchanged. Thus, at any date $t \geq 0$, given the contemporaneous quality level $A_{j i}(t)$ and the number of scientists $L_{j i}(t)$, it can be established that the expected instantaneous rise in $A_{j i}(t)$ is given by the standard law of motion

$$
\dot{A}_{j i}(t)=\gamma A_{j i}(t) \eta_{j} L_{j i}(t), \forall j=c, d, \forall i \in[0,1]
$$

with $A_{j i}(0)=A_{j k}(0)$ for all $j=c, d$ and any $i, k \in[0,1]$.

The arrival of innovation-generated intermediate-good versions raises the issue of how property rights are defined; an issue at the core of modern economic theories accounting for private incentives to perform R\&D. Acemoglu et al. (2012) assume that any successful scientist is given a temporary monopoly right over the benefit derived from sales of the intermediate-good version generated by her innovation. In their discrete-time framework, they assume that such patents are only enforced over the smallest definable unit of time, that is a period. In the long-run perspective of growth theory, the normalization is meaningful. ${ }^{13}$ Most importantly, it is particularly convenient as it rules out the possibility, technically unmanageable in such models, that an innovation occurs in one sector while rights over the last intermediate-good version are still being enforced.

The assumption has a clear counterpart in our continuous-time model, implying that any patent is only enforced at the very date when the innovation occurs. Even when time is continuous, the crucial existence of quasi-rents which motivate R\&D investments is compatible with the normalization. As we shall see, the assumption thus reproduces the standard structure of endogenous innovation processes in the simplest possible manner. On the other hand, the innovation process need not be continuous. To avoid discontinuity in intermediate goods' pricing, however, we strictly follow Acemoglu et al. (2012): when no scientist is suc-

\footnotetext{
${ }^{13}$ Since in practice intellectual property rights are enforced for a finite-time duration, the assumption is arguably stronger than the often-made alternative one that rights last forever.
} 
cessful in one sector, the monopoly right survives and is randomly allocated to any potential entrepreneur, who then exploits the last intermediate-good version.

In either case, at each date $t \geq 0$, there is always a single intermediate good $x_{j i}(t)$ of quality level $A_{j i}(t)$, which is produced by a monopoly, according to the linear production function

$$
x_{j i}(t)=\frac{1}{\psi} y_{j i}(t), j=c, d, i \in[0,1],
$$

with $\psi>0$ and where $y_{j i}(t)$ is an amount of final good.

The preferences of the representative, infinitely-lived household, are represented by the intertemporal utility function

$$
U=\int_{0}^{+\infty} \ln (C(t)) e^{-\rho t} d t
$$

where $\rho$ is a constant discount rate. ${ }^{14}$ For our purpose, the intertemporal elasticity of substitution does not play any crucial role; the logarithmic form of the felicity function thus normalizes this elasticity to unity for simplicity.

Households are endowed with the constant flow $L>0$ of labor energy; each unit of labor is competitively supplied by one scientist to the two competing R\&D sectors. Normalizing the mass of scientists to unity, it must be that

$$
\int_{0}^{1} L_{c i}(t) d i+\int_{0}^{1} L_{d i}(t) d i=1, \forall t \geq 0 .
$$

Last, the final good produced at each date $t \geq 0$ is either used for consumption or for the production of clean and dirty intermediate goods:

$$
Y(t)=C(t)+\int_{0}^{1} y_{c i}(t)+\int_{0}^{1} y_{d i}(t), \forall t \geq 0 .
$$

\footnotetext{
${ }^{14}$ An explicit modeling of environmental damages would have raised the issue of how households are affected by those damages. The rest of the analysis would have remained unchanged with separable damages, under which the marginal utility solely depends on consumption.
} 


\section{Input Demands by Production Sectors}

As is usual, we choose the final good as the numeraire good; its price is normalized to unity. In the rest of the paper, $p_{j i}(t), p_{Q}(t), p_{R}(t)$ will respectively denote the price of the intermediate good $i \in[0,1]$ used in sector $j=c, d$, the price of the clean renewable resource and the price of the dirty non-renewable resource. Because they are competitive, the final sector and the clean and dirty production sectors can be aggregated without loss of generality so that their joint problem simply consists in the maximization of their total profit

$$
\begin{aligned}
\pi_{Y}(t) & =\left[Y_{c}(t)^{(\varepsilon-1) / \varepsilon}+Y_{d}(t)^{(\varepsilon-1) / \varepsilon}\right]^{\varepsilon /(\varepsilon-1)} \\
& -\sum_{j=c, d} \int_{0}^{1} p_{j i}(t) x_{j i}(t) d i-p_{Q}(t) Q-p_{R}(t) R(t),
\end{aligned}
$$

where prices are taken as parameters.

\subsection{Resource Demand}

As one can anticipate in light of Sinn's (2008) analysis, resource demand will turn out to play the most fundamental role in the determination of the speed at which the resource is extracted.

The first-order condition for the choice of resource input $R(t)$ writes $p_{R}(t)=(1-$ $\alpha) Y(t)^{1 / \varepsilon} Y_{d}(t)^{(\varepsilon-1) / \varepsilon} / R(t)$, which equalizes the marginal productivity of the resource input

to its price. Using (1), the marginal productivity of the resource can be expressed in such a way that

$$
p_{R}(t)=\frac{(1-\alpha) Y(t)}{R(t)} \frac{1}{\left(Y_{c}(t) / Y_{d}(t)\right)^{(\varepsilon-1) / \varepsilon}+1},
$$

which must be interpreted using the concept of conditional factor demand: precisely, the resource productivity as given by the right-hand side of (11) is the inverse resource demand function, conditional upon the amount of final output $Y(t)$.

As (11) shows, the conditional resource demand function only depends on the relative contribution $Y_{c}(t) / Y_{d}(t)$ of sectors $c$ and $d$ to the economy, yet in a way that involves sectors' degree of substitutability/complementarity $\varepsilon$. Smulders and Di Maria (2012) recently 
pointed at the crucial and intricate channels by which technological change determines inputs demand. In a multi-sector context, formula (11) further shows the role of the relative contribution of sectors: for a given output level, the relative contribution of sectors completely summarizes the determinants of resource marginal productivity and thus of total resource demand. The rest of the analysis will examine how the relative sectors' contribution is affected by $R \& D$ policies, thus making the following proposition a central result of the paper.

Proposition 1 A rise in the relative contribution of the clean sector to the economy causes the conditional resource demand function

ı) to decrease if the clean and dirty sectors are (gross) substitutes $(\varepsilon>1$ ) or

ı) to increase if these sectors are (gross) complements $(\varepsilon<1)$.

\subsection{Intermediate Good Quantities}

On the one hand, the first-order conditions for the choice of quantities $x_{c i}(t)$ and $x_{d i}(t)$ of clean and dirty intermediate goods maximizing (10) are

$$
\begin{aligned}
& p_{c i}(t)=\alpha Y(t)^{1 / \varepsilon} Y_{c}(t)^{-1 / \varepsilon}\left[\frac{Q A_{c i}(t)}{x_{c i}(t)}\right]^{1-\alpha} \text { and } \\
& p_{d i}(t)=\alpha Y(t)^{1 / \varepsilon} Y_{d}(t)^{-1 / \varepsilon}\left[\frac{R(t) A_{d i}(t)}{x_{d i}(t)}\right]^{1-\alpha}
\end{aligned}
$$

which give the production sectors' inverse demands for intermediate goods.

On the other hand, our assumptions imply the standard property that at each date $t \geq 0$, all intermediate goods $x_{j i}(t), j=c, d, i \in[0,1]$, are monopolistically supplied. By (6), producing a quantity $x_{j i}(t)$ of intermediate good requires an amount $\psi x_{j i}(t)$ of final good. The profit derived from this activity thus writes

$$
\pi_{j i}(t)=x_{j i}(t)\left[p_{j i}(t)-\psi\right]
$$

where $p_{j i}(t)$ is given by (12) and (13) because monopolies integrate the sensitiveness of the demand they face. In this context, monopoly prices $p_{j i}(t)$ exhibit a mark-up above the 
marginal cost $\psi$,

$$
p_{j i}(t)=\frac{\psi}{\alpha}, \forall j=c, d, \forall i \in[0,1]
$$

and turn out to be time-invariant as well as independent of the sector $j=c, d$ to which they are dedicated and of the type of intermediate good $i \in[0,1]$.

As a result, inverse demand functions (12) and (13) imply that the equilibrium quantity of intermediate good $x_{j i}(t)$ proportionally depends on the quality level $A_{j i}(t)$ in a way that is independent of the type of intermediate $\operatorname{good} i \in[0,1]$. Making use of this property, Appendix A combines the relative contribution of the clean sector $Y_{c}(t) / Y_{d}(t)$ obtained from production functions (3) and (4) with the relative marginal productivity derived from (12) and (13) and shows the following identity:

$$
\frac{Y_{c}(t)}{Y_{d}(t)}=\left[\frac{x_{c}(t)}{x_{d}(t)}\right]^{\varepsilon /(\varepsilon-1)},
$$

where $x_{c}(t) \equiv \int_{0}^{1} x_{c i}(t) d i$ and $x_{d}(t) \equiv \int_{0}^{1} x_{d i}(t) d i$ denote the average quantities of intermediate goods respectively used in the clean and dirty production sectors. ${ }^{15}$

As (16) shows, at the production sector's optimum, changes in the relative contribution of the clean sector are completely summarized by changes in the relative use of intermediate goods by this sector. This is the message of the following lemma which will later turn out to be particularly useful.

Lemma 1 In equilibrium, the relative contribution of the clean sector to the economy ı) increases with the relative use of clean intermediate goods if the clean and dirty sectors are (gross) substitutes $(\varepsilon>1)$ and

ı) decreases with it if these sectors are (gross) complements $(\varepsilon<1)$.

\footnotetext{
${ }^{15}$ The notations are introduced here for simplicity. It will shortly turn out to be true that $x_{j}(t)=x_{j i}(t)$, for all $j=c, d$, for all $i \in[0,1]$.
} 


\section{R\&D-Support Policies and Directed Technical Change}

This section investigates how $R \& D$ and intermediate activities are affected by $R \& D$-support policies. For that purpose, we first establish how economic resources are allocated to competing $R \& D$ sectors in equilibrium.

When an innovation occurs at date $t \geq 0$, giving rise to a new, more productive type of intermediate good $x_{j i}(t), j=c, d, i \in[0,1]$, the innovator is entitled with an exclusive right over the profits $\pi_{j i}(t)$ immediately derived from the sales of the new intermediate good. The most basic and meaningful way to support R\&D activities is to subsidize innovators' profits. Let $\lambda_{j}(t) \geq 1$ be the continuously time-varying subsidy factor ${ }^{16}$ applied to any innovator's profit in sector $j=c, d$. Hence, the inclusive-of-subsidy benefit from innovating is ${ }^{17}$

$$
V_{j i}(t)=\lambda_{j}(t) \pi_{j i}(t)
$$

where it follows from the analysis of the previous section (formula (14) with equilibrium price (15)) that $\pi_{j i}(t)$ can be expressed as a parametric function of $x_{j i}(t)$ only:

$$
\pi_{j i}(t)=\frac{(1-\alpha) \psi}{\alpha} x_{j i}(t)
$$

At each date $t \geq 0, L_{j i}(t)$ scientists are dedicated to improving the quality level of intermediate good $i \in[0,1]$ used in sector $j=c, d$, each having the instantaneous probability

\footnotetext{
${ }^{16} \lambda_{j}(t)=1+\tau_{j}(t)$, where $\tau_{j}(t) \geq 0$ is the unique subsidy rate corresponding to the subsidy factor $\lambda_{j}(t)$. All along the analysis, $\tau_{j}(t)$ need not be made explicit.

${ }^{17}$ Our modeling choice to follow Acemoglu et al. (2012) in assuming the duration of patents to be normalized to the smallest definable unit of time makes expression (17) much simpler than in traditional treatments where the benefit from innovating not only involves contemporaneous monopoly profits, but also the discounted stream of future expected ones. As announced in the introduction, the simplification does not imply any departure from the regular endogenous-growth mechanism where prospects of quasi-rents motivate $\mathrm{R} \& \mathrm{D}$ investments. In fact, the rest of the analysis would have remained formally the same if patents were assumed to be enforced for a long time, while the rate at which profits are discounted were taken as given. The proof goes as follows. Let parameter $\theta(t)$ be this given rate of discount, which consists of the interest rate and possibly of an instantaneous risk of patents' erosion in the form described by Barro and Sala-i-Martin (1995, Ch. 6). Then, expression (17) would become $V_{j i}(t)=\int_{t}^{+\infty} \lambda_{j}(s) \pi_{j i}(s) e^{-\int_{t}^{s} \theta(u) d u} d s$. Differentiating with respect to time by Leibniz rule would give $\dot{V}_{j i}(t) / V_{j i}(t)-\theta(t)=-\left(\lambda_{j}(t) \pi_{j i}(t)\right) / V_{j i}(t)$, for the two sectors $j=c, d$. However, the analysis would only change to this extent: the R\&D profit (19) as well as the free-entry condition (20) would still apply to both sectors. Because the latter implies the growth rate of $V_{j i}(t)$ to be the same in the two sectors, the fundamental no-arbitrage equation (21) as well as the rest of the analysis would then hold in the same manner as under our simplifying assumption.
} 
$\eta_{j}$ of being the successful innovator. For any $j=c, d$, and any $i \in[0,1]$, the total profit from R\&D activities thus writes

$$
\pi_{R \& D j i}(t)=\eta_{j} L_{j i}(t) V_{j i}(t)-w(t) L_{j i}(t)
$$

where $w(t)$ is the wage rate and $V_{j i}(t)$ is given by $(17)$.

As is well known, it is theoretically possible in directed-technical-change models that $\pi_{R \& D j i}(t)$ be strictly negative for any strictly positive $L_{j i}(t)$, so that innovations do not occur in all sectors simultaneously (see e.g. Acemoglu, 2008, Ch. 15).

Empirical evidence shows that specific R\&D activities currently take place in resource and non-resource sectors (Aghion et al., 2011). As Appendix B shows, such interior allocation of research efforts arises, either regardless of $R \& D$-support policies that are the object of this paper, or as a consequence of these policies. The appendix assumes that the economy starts from the initial corner equilibrium of Acemoglu et al. (2012) where innovations exclusively occur in the resource sector; as a result of R\&D-support policies that sufficiently favor the clean non-resource R\&D sector, the economy's equilibrium becomes interior in finite time and remains so all along its long-run path.

Thus in the rest of the analysis, we focus on the long-run paths where the allocation of labor to R\&D sectors is interior: labor entry is always profitable in the two, resource and non-resource, sectors; in any such equilibria, arbitrage possibilities result in the standard free-entry condition which applies to the clean and to the dirty sectors in a similar manner. From (19), it must be that

$$
\eta_{c} V_{c i}(t)=\eta_{d} V_{d i}(t)=w(t), \forall i \in[0,1]
$$

which means that the marginal productivity of scientists is equalized across R\&D sectors.

The above free-entry condition has two main implications. The first one amounts to a simplification. According to $(20)$, the net benefit of innovating $V_{j i}(t)$ may only differ across sectors $j=c, d$ by the probability parameters $\eta_{c}$ and $\eta_{d}$, but will not depend on the type of intermediate good $i \in[0,1]$ that the associated innovation improves. By (17) and (18), the 
same property applies to profits $\pi_{j i}(t)$ and to intermediate good quantities $x_{j i}(t)$. In the rest of the analysis, we will make use of the notations $V_{j}(t)=V_{j i}(t)$ and $\pi_{j}(t)=\pi_{j i}(t)$, while our earlier definition $x_{j}(t) \equiv \int_{0}^{1} x_{j i}(t) d i$ now becomes the equilibrium identity $x_{j}(t)=x_{j i}(t)$, for any $j=c, d$ and $i \in[0,1]$.

The second implication of (20) is an essential piece of the paper's demonstration. The freeentry condition tells that the relative inclusive-of-subsidy benefit of innovating $V_{c}(t) / V_{d}(t)$ is determined in a way that is irrespective of subsidy rates $\lambda_{c}(t)$ and $\lambda_{d}(t)$. Recalling that by $(17)$, the net value $V_{j}(t)$ only depends on the subsidy rate $\lambda_{j}(t)$ and on profit $\pi_{j}(t)$, we obtain the more enlightening condition

$$
\eta_{c} \lambda_{c}(t) \pi_{c}(t)=\eta_{d} \lambda_{d}(t) \pi_{d}(t)
$$

which shows that in absence of arbitrage, any support to the clean sector by say an increase in the relative subsidy $\lambda_{c}(t) / \lambda_{d}(t)$ is necessarily compensated by a change in relative profits $\pi_{c}(t) / \pi_{d}(t)$ of opposite direction.

No-arbitrage conditions are the keystone of equilibria in economies with competing investment possibilities. While absent in one-sector endogenous-growth models, directed-technicalchange models reveal that such conditions prevail at the R\&D stage, that returns to investing in $R \& D$ must equalize across all active $R \& D$ sectors. Thus, taking a full account of the process by which specific R\&D investments are implemented as a response to economic incentives, clearly yields the above result that may otherwise be not so intuitive. Another surprising aspect of the demonstration may be that it applies regardless of the effect of R\&D-support subsidies on the actual technological bias between the two sectors.

Keeping in mind from (18) that profits of the intermediate sectors can be expressed as functions of intermediate good quantities only, we immediately obtain the following lemma.

Lemma 2 In any interior equilibrium allocation, the relative use of clean intermediate goods always decreases with the relative subsidy to clean $R \& D$ activities.

Lemma 2 provides an essential result of the paper. Shortly below, it will nicely combine 
with Lemma 1 and with Proposition 1 so as to determine the effect of R\&D-support policies on resource demand.

\section{R\&D-Support Policies and the Green Paradox}

\subsection{Transmission Channel}

The first part of this section aims at summarizing the effect of R\&D-support policies on resource demand. For that, we can rely on the results established earlier. On the one hand, Lemma 2 states the effect of R\&D-support policies on the relative use of intermediate goods by the clean and dirty sectors. On the other hand, Lemma 1 tells us that the relative use of intermediate goods completely summarizes the relative contribution of the clean and dirty sectors. The combination of Lemma 1 with Lemma 2 immediately yields the following proposition on the effect of R\&D-support policies on the relative contribution of the clean and dirty sectors.

Proposition $2 A$ rise in the relative subsidy to clean $R \& D$ activities causes the equilibrium relative contribution of the clean sector to the economy

ı) to decrease if the clean and dirty sectors are (gross) substitutes $(\varepsilon>1)$ and

ı) to increase if these sectors are (gross) complements $(\varepsilon<1)$.

Formally speaking, Proposition 2 results from the combination of identity (16) that $Y_{c}(t) / Y_{d}(t)=\left[x_{c}(t) / x_{d}(t)\right]^{\varepsilon /(\varepsilon-1)}$, with condition $(21)$ that $\eta_{c} \lambda_{c}(t) \pi_{c}(t)=\eta_{d} \lambda_{d}(t) \pi_{d}(t)$, making use of (18), which implies relative profits $\pi_{c}(t) / \pi_{d}(t)$ to be equal to relative intermediate quantities $x_{c}(t) / x_{d}(t)$. Hence, in any interior equilibrium, we have

$$
\frac{Y_{c}(t)}{Y_{d}(t)}=\left[\frac{\lambda_{d}(t) \eta_{d}}{\lambda_{c}(t) \eta_{c}}\right]^{\varepsilon /(\varepsilon-1)} .
$$

Come back now to resource demand. Substituting (22) in expression (11) gives the conditional inverse resource demand

$$
p_{R}(t)=\frac{(1-\alpha) Y(t)}{R(t)} \Lambda(t)
$$


where, for notational simplicity, we make use of the policy index $\Lambda(t)$ defined as

$$
\Lambda(t) \equiv \frac{1}{1+\frac{\lambda_{d}(t) \eta_{d}}{\lambda_{c}(t) \eta_{c}}}
$$

The index $\Lambda(t)$ is a basic measure of the relative support to clean R\&D activities. For what follows, it only matters that $\Lambda(t)$ is monotonically increasing in the relative subsidy to clean R\&D $\lambda_{c}(t) / \lambda_{d}(t)$. Thus, equation (23) expresses the conditional resource demand, no longer as a function of $Y_{c}(t) / Y_{d}(t)$ as in (11), but directly as a function of R\&D-support policies.

Neither formula (23) nor definition (24) involves the elasticity of substitution $\varepsilon$. Thus it turns out that, taking the output-level condition $Y(t)$ as given in formula (23), a rise in the relative clean-R\&D subsidy causes resource demand to increase, regardless of the pattern of substitutatility/complementarity between the two sectors. Indeed, although the role of the sectoral elasticity of substitution $\varepsilon$ appears crucial in Proposition 2 (relation between R\&D-support policies and sectors' contribution) and in Proposition 1 (relation between sectors' contribution and resource demand), this role completely vanishes when the results combine to determine the overall effect of policies on resource demand. To sum up, the final message arising from Propositions 1 and 2 is independent of the sectors' degree of substitutability/complementarity. Indeed a rise in the relative clean-R\&D subsidy always causes resource demand to increase.

So far, results have been established as static effects holding at each date $t$ of the time set $[0,+\infty)$. Since the resource is non-renewable, its extraction pattern cannot be directly deduced from the above static analysis; similarly, the effects of R\&D-support policies on the extraction speed cannot be deduced from the above static result arising from Propositions 1 and 2. The next - and last - section extends the above result from the static frame of the previous sections to its dynamic counterpart.

\subsection{Impact of R\&D-Support Policies on the Resource Extraction Pattern}

Extending the above static results summarized by formula (23) so as to derive the effects of R\&D-support policies on the speed of resource extraction further requires taking into 
account intertemporal decisions which determine the dynamic equilibrium.

On the one hand, households' consumption/saving arbitrage determines the growth rate of final output $Y(t)$ in (23). The maximization of the intertemporal utility objective (7), subject to any intertemporal budget constraint arising under a perfect financial market, yields the standard Ramsey-Keynes condition: $g_{C}(t)=r(t)-\rho$, where $r(t)$ is the rate of interest endogenously determined on the financial market, and where, as in the rest of the paper, the symbol $g$ with a variable subscript denotes the growth rate of this variable. As Appendix C shows, although consumption is not the exclusive use of final output, the Ramsey-Keynes condition applying to the former also dictates the law of motion of the latter. Formally,

$$
g_{Y}(t)=r(t)-\rho, \forall t \geq 0
$$

must hold in any dynamic equilibrium.

On the other hand, the same assumption that there exists a perfect financial market implies that the standard rule of Hotelling must hold, meaning that any unit of reserves must fetch the same revenue in present value. Under our simplifying, although conventional in growth models, assumption that extraction is costless, the rule writes

$$
g_{p_{R}}(t)=r(t), \forall t \geq 0
$$

which must be satisfied in any dynamic equilibrium.

Consider finally the conditional expression of resource demand (23) and its dynamic implications. Log-differentiating both sides and making use of the Keynes-Ramsey rule (25) and of the Hotelling rule (26), the expression of the speed of extraction immediately follows,

$$
g_{R}(t)=g_{\Lambda}(t)-\rho,
$$

where the effects of the interest rate on $g_{Y}(t)$ and $g_{p_{R}}(t)$ have canceled out.

We are left with the following statement which concludes our analysis.

Proposition 3 In any dynamic interior equilibrium, a gradual rise of the relative subsidy to clean $R \& D$ activities over time induces resource extraction to be less rapid, irrespective of the pattern of substitutability/complementarity between the clean and dirty production sectors. 
This final result should be interpreted through the lens of the preceding static messages of Propositions 1 and 2. Their combination and their dynamic extension tells the following. A gradual rise of the relative subsidy to clean $R \& D$, whatever its other dynamic effects on the economy's structure, will always contribute to gradually increasing the demand for the resource. The rest is standard: the resulting rise in future demand relative to early demand leads market forces to exploit less resource at early dates and so more at distant ones.

\section{Conclusion}

Technical improvements in the production of clean substitutes to dirty non-renewable resources have been considered tantamount to policies that reduce the demand for those resources (e.g. Sinn, 2008). In this context, such technical improvements, whether expected to take place in the long-term future, or gradually induced by more-and-more stringent R\&Dsupport policies, would cause an undesirable green paradox phenomenon. The pessimistic argument has been made in parsimonious models, either adopting a partial-equilibrium approach taking the technology as exogenous, or assuming a single resource-consuming sector.

This paper shows that the conjecture is wrong. Borrowing standard assumptions from the modern-economic-growth literature, the analysis takes a full account of the endogenous directed R\&D process by which productivity is specifically enhanced in clean renewableresource and dirty non-renewable-resource sectors, and of the policy instruments by which such $R \& D$ activities can be promoted. When the allocation of labor efforts to R\&D sectors is interior, as is shown by empirical evidence, we find that a gradual support to R\&D activities aimed to improve productivity in clean sectors increases, among other effects, the long-run

productivity and thus the demand for the non-renewable resource. From a policy perspective, the message delivered by the analysis is much more optimistic than the aforementioned conjecture. Supporting clean R\&D sectors does not cause a green paradox phenomenon. 


\section{APPENDICES}

\section{A Proof of identity (16)}

Substituting $p_{c i}(t)=p_{d i}(t)=\psi / \alpha$ from (15) into equalities (12) and (13) and dividing the left and right sides of the latter by both sides of the former yield the following expression which gives the relative contribution of sectors

$$
\left(\frac{Y_{c}(t)}{Y_{d}(t)}\right)^{1 / \varepsilon}=\left(\frac{Q}{R(t)}\right)^{1-\alpha}\left(\frac{A_{c i}(t) / x_{c i}(t)}{A_{d k}(t) / x_{d k}(t)}\right)^{1-\alpha}, \forall i, k \in[0,1], \forall t \geq 0 .
$$

In this identity, the ratios $A_{j i}(t) / x_{j i}(t)$ are independent of $i \in[0,1]$ for each $j=c, d$ as shown by (12) and (13) after the substitution of $p_{j i}(t)$ from (15).

The latter property implies that (3) and (4) can be rewritten with $\int_{0}^{1} A_{j i}(t)^{1-\alpha} x_{j i}(t)^{\alpha} d i=$ $\left(A_{j i}(t) / x_{j i}(t)\right)^{1-\alpha} \int_{0}^{1} x_{j i}(t) d i$ where by definition $\int_{0}^{1} x_{j i}(t) d i \equiv x_{j}(t)$. Dividing the obtained expressions of equalities (3) and (4) by each other yields

$$
\frac{Y_{c}(t)}{Y_{d}(t)}=\left(\frac{Q}{R(t)}\right)^{1-\alpha}\left(\frac{A_{c i}(t) / x_{c i}(t)}{A_{d k}(t) / x_{d k}(t)}\right)^{1-\alpha} \frac{x_{c}(t)}{x_{d}(t)}, \forall i, k \in[0,1], \forall t \geq 0 .
$$

Finally, dividing (A.1) by (A.2) immediately gives identity (16).

\section{B Convergence to an Interior Allocation of Scientists to the two R\&D Sectors}

This appendix shows how, in the context under study, the economy converges to, and remains in, a long-run interior equilibrium where the two R\&D sectors are simultaneously active.

On the one hand, (17) and (18) show that the relative benefit from innovating depends on the relative use of intermediate goods $x_{c}(t) / x_{d}(t)$. On the other hand, the relative use of intermediates is determined by the relative input demands of production sectors (see (16)), which depend on the dynamics of innovation. Thus in the following analysis, a central role will be played by the relative use of intermediate goods, that we denote as follows for notational simplicity:

$$
u(t) \equiv \frac{x_{c}(t)}{x_{d}(t)}, \forall t \geq 0
$$

where $x_{j}(t) \equiv \int_{0}^{1} x_{j i}(t) d i, j=c, d$.

The first part of the appendix establishes the general motion of $u(t)$ that results from input demands, and thus from research efforts. The second part will characterize interior equilibria. In presence of $R \& D$-support policies that favor clean $R \& D$ activities, as is the case in the context of this paper, the economy may converge to an interior allocation of R\&D efforts, regardless of whether the clean and dirty production sectors are (gross) substitutes $(\varepsilon>1)$ or complements $(\varepsilon<1)$. 


\section{Dynamics of the relative use of intermediate goods $u(t)$}

This section characterizes the dynamics of $u(t)$ that results from input demands. For that, it takes R\&D efforts $L_{c i}(t)$ and $L_{d i}(t)$ for any $i \in[0,1]$ as given: no assumption is required on whether the allocation of those efforts is interior or not.

Dividing the demand for intermediate goods (12) and (13) by each other and substituting the intermediate monopoly prices (15), one obtains (A.1). The property (see Appendix A) that the ratios $A_{j i}(t) / x_{j i}(t)$ are independent of $i \in[0,1]$ for each $j=c, d$ implies that $A_{j i}(t) / x_{j i}(t)=A_{j}(t) / x_{j}(t)$, with $x_{j}(t) \equiv \int_{0}^{1} x_{j i}(t) d i$ and where $A_{j}(t) \equiv \int_{0}^{1} A_{j i}(t) d i$ denotes the average quality level of intermediates of type $j=c, d$. Thus, (A.1) may rewrite

$$
\left(\frac{Y_{c}(t)}{Y_{d}(t)}\right)^{1 / \varepsilon}=\left(\frac{Q}{R(t)}\right)^{1-\alpha}\left(\frac{A_{c}(t) / x_{c}(t)}{A_{d}(t) / x_{d}(t)}\right)^{1-\alpha}, \forall t \geq 0 .
$$

Further using the input-output relations encompassed in (16) to substitute $Y_{c}(t) / Y_{d}(t)$ in the latter identity, and rearranging, yield

$$
u(t)=\left(\frac{Q A_{c}(t)}{R(t) A_{d}(t)}\right)^{\frac{(1-\alpha)(\varepsilon-1)}{(1-\alpha) \varepsilon+\alpha}}
$$

that must hold in any equilibrium, at all dates $t \geq 0$.

To derive the dynamic behavior of $u(t)$, it will be useful to first examine the variables that enter the right-hand side of (B.1).

Along this appendix, we will focus on symmetric equilibria where, in each R\&D sector $j=c, d$, scientists are evenly allocated over the range $i \in[0,1]$. Specifically, for each $j=c, d$, $L_{j i}(t)$ is assumed independent of $i \in[0,1]$. This simplifying assumption is a standard one in similar Schumpeterian-growth models (see Cozzi et al., 2007, on the assumption and on some foundations). Further below, we shall see that the within-sector symmetry will turn out to arise endogenously in any interior equilibrium. Thus we can define $L_{j}(t)=L_{j i}(t)$, which is also the total number of scientists $\int_{0}^{1} L_{j i}(t) d i$ employed in the R\&D sector $j=c, d$. In this context, summing over the range $i \in[0,1]$ all equations like (5) and making use of the variable $A_{j}(t) \equiv \int_{0}^{1} A_{j i}(t) d i$ to denote the average quality level in each sector $j=c, d$, we obtain the average quality levels' laws of motion

$$
g_{A_{j}}(t)=\gamma \eta_{j} L_{j}(t), j=c, d, \text { for all } L_{j}(t) \in[0,1],
$$

where the symbol $g$ with a variable subscript denotes the growth rate of the variable. We are going to make use of this equation shortly below.

With (16), the resource demand equation (11) immediately writes

$$
p_{R}(t)=\frac{(1-\alpha) Y(t)}{R(t)} \frac{1}{1+u(t)} .
$$

Log-differentiating both sides of (B.3), making use of the Keynes-Ramsey rule (25) and of the Hotelling rule (26) and simplifying, one obtains the following expression for the extraction growth rate:

$$
g_{R}(t)=\frac{-\dot{u}(t)}{1+u(t)}-\rho
$$


that we are also going to use shortly.

Log-differentiating (B.1) and substituting the growth rates of $A_{c}(t), A_{d}(t)$ and $R(t)$ by their expressions in (B.2) and (B.4), we obtain

$$
\frac{\dot{u}(t)}{u(t)}=\frac{(1-\alpha)(\varepsilon-1)}{(1-\alpha) \varepsilon+\alpha}\left(\rho+\gamma \eta_{c} L_{c}(t)-\gamma \eta_{d} L_{d}(t)+\frac{\dot{u}(t)}{1+u(t)}\right) .
$$

Rearranging and simplifying yield the following expression for the growth rate of variable $u(t)$ :

$$
g_{u}(t)=\frac{(1-\alpha)(\varepsilon-1)(1+u(t))}{(1-\alpha) \varepsilon+\alpha+u(t)}\left(\rho+\gamma \eta_{c} L_{c}(t)-\gamma \eta_{d} L_{d}(t)\right)
$$

where one can verify that $\frac{(1-\alpha) \varepsilon+\alpha+u(t)}{(\varepsilon-1)(1+u(t))}=\frac{\varepsilon}{\varepsilon-1}-\frac{\alpha+u(t)}{1+u(t)}$. Finally, in any equilibrium, the law of motion of the relative use of intermediates may be characterized as the following function of research efforts in the two R\&D sectors $L_{c}(t)$ and $L_{d}(t)$ :

$$
g_{u}(t)=\frac{1-\alpha}{\frac{\varepsilon}{\varepsilon-1}-\frac{\alpha+u(t)}{1+u(t)}}\left(\rho+\gamma \eta_{c} L_{c}(t)-\gamma \eta_{d} L_{d}(t)\right) .
$$

\section{Interior allocation of R\&D efforts}

Not all values of the relative use of intermediates $u(t)$ are compatible with the realization of an interior equilibrium in which the two $R \& D$ sectors are active. For strictly positive research efforts $L_{j}(t)$ to be made in the two sectors $j=c, d$, the no-arbitrage condition (21) must be satisfied. With (18), that means that $u(t)$ must be at the following level that we denote $\bar{u}(t)$ :

$$
u(t)=\bar{u}(t) \equiv \frac{\eta_{d} \lambda_{d}(t)}{\eta_{c} \lambda_{c}(t)}
$$

which negatively depends on the relative subsidy $\lambda_{c}(t) / \lambda_{d}(t)$ to clean R\&D activities. Such relative subsidies to clean $R \& D$ activities that are rising over time are the object of this paper; thus $\bar{u}(t)$ should be considered to be strictly decreasing over time, from some initial level $\bar{u}(0)=\frac{\eta_{d} \lambda_{d}(0)}{\eta_{c} \lambda_{c}(0)}$ to some arbitrarily low value in the long run. The analysis further assumes that subsidy factors $\lambda_{c}(t)$ and $\lambda_{d}(t)$ are evolving continuously over time; hence so does $\bar{u}(t)$.

When $u(t)$ is not equal to $\bar{u}(t)$, the economy does not feature an interior allocation of labor efforts to the two R\&D sectors $j=c, d$. In light of the analysis of Section 4 , when $u(t)>\bar{u}(t)$, then $L_{d}(t)=0$ and $L_{c}(t)=1-L_{d}(t)=1$, whereas when $u(t)<\bar{u}(t), L_{d}(t)=1$ and $L_{c}(t)=0$. Only when the economy remains at the intersection of $u(t)$, as determined by (B.6), with $\bar{u}(t)$, the economy can be in an interior equilibrium.

\section{Convergence towards an interior allocation of R\&D efforts}

In the sequel, following Acemoglu et al. (2012), we assume that the economy is initially in the corner equilibrium where there is no activity in the clean R\&D sector, i.e. $L_{c}(0)=0$ and $L_{d}(0)=1$. This equilibrium is only compatible with $u(0)$ being lower than $\bar{u}(0)$. For all dates $t>0, \bar{u}(t)$ is continuously decreasing over time, while $u(t)$ continuously evolves 
according to (B.6). As long as $u(t)$ and $\bar{u}(t)$ do not intersect, $u(t)<\bar{u}(t)$ so that the economy remains in the initial corner equilibrium where $L_{c}(t)=0$ and $L_{d}(t)=1$.

Then the term into parentheses in (B.6) reduces to $\rho-\gamma \eta_{d}$; it is standard to consider this term negative to mean that the $R \& D$ technology is sufficiently effective. The other term on the right-hand side of (B.6) is that of the denominator $\varepsilon /(\varepsilon-1)-(\alpha+u(t)) /(1+u(t))$. As concerns its sign, two cases must be distinguished according to whether the two sectors are (gross) complements or substitutes.

ı) Case $\varepsilon<1$ :

In this case, $\varepsilon /(\varepsilon-1)$ is negative, and so is $\varepsilon /(\varepsilon-1)-(\alpha+u(t)) /(1+u(t))$, for any $u(t) \geq 0$. Since the term of (B.6) into parentheses is also negative as long as $u(t)$ remains lower than $\bar{u}(t), g_{u}(t)$ is strictly positive. By continuity of $u(t)$ and $\bar{u}(t)$, they must intersect in finite time, as illustrated in Figure 1; the intersection date $T \geq 0$ occurs all the earlier as $\bar{u}(t)$ is steep, i.e. as policies support clean R\&D activities rapidly. After $T$, there is an interior equilibrium $u(t)=\bar{u}(t)$ in which the economy remains in the long run. We shall characterize this equilibrium further below.

थ) Case $\varepsilon>1$ :

In this case, $\varepsilon /(\varepsilon-1)>1$ while $(\alpha+u(t)) /(1+u(t))<1$ for any $u(t) \geq 0$. Thus $\varepsilon /(\varepsilon-1)-(\alpha+u(t)) /(1+u(t))$ is positive in (B.6). In the same equation, the term into parentheses is negative as long as $u(t)$ does not intersect $\bar{u}(t)$. Hence, $u(t)$ is now decreasing over time as long as it remains lower than $\bar{u}(t)$. There may be no intersection between $u(t)$ and $\bar{u}(t)$, in which case the economy remains along an "environmental disaster" path (Acemoglu et al., 2012). For our purpose, this uninteresting case is assumed away: specifically, we assume that $\bar{u}(t)$ is sufficiently decreasing over time, as is the case when the relative subsidy to clean R\&D activities rise rapidly, so as to ensure that $u(t)$ intersects $\bar{u}(t)$ in finite time. Like when $\varepsilon<1$, there is an interior equilibrium $u(t)=\bar{u}(t)$ in which the economy remains at all dates following the intersection date $T \geq 0$ (Figure 2).

In either case, the interior equilibrium in which the economy remains at all dates $t \geq T$ can be characterized as follows. In this equilibrium, $u(t)=\bar{u}(t)$ for all $t \geq T$, so that $L_{c}(t)>0$ and $L_{d}(t)>0$ must satisfy (B.5) where $u(t)$ is replaced by $\bar{u}(t)$. The values $L_{c}(t)$ and $L_{d}(t)$ in this equilibrium are thus defined as the following function of the exogenous policy variable $\bar{u}(t)$ :

$$
\frac{\dot{\bar{u}}(t)}{\bar{u}(t)}=\frac{(1-\alpha)(\varepsilon-1)}{(1-\alpha) \varepsilon+\alpha}\left(\rho+\gamma \eta_{c} L_{c}(t)-\gamma \eta_{d} L_{d}(t)+\frac{\dot{\bar{u}}(t)}{1+\bar{u}(t)}\right) .
$$

Rearranging adequately and replacing $L_{d}(t)$ by $1-L_{c}(t)$ yield the expression

$$
L_{c}(t)=\frac{1}{\gamma\left(\eta_{c}+\eta_{d}\right)}\left(\gamma \eta_{d}-\rho-\frac{\dot{\bar{u}}(t)}{1+\bar{u}(t)}+\frac{(1-\alpha) \varepsilon+\alpha}{(1-\alpha)(\varepsilon-1)} \frac{\dot{\bar{u}}(t)}{\bar{u}(t)}\right), \forall t \geq T .
$$

The above equilibrium exists as long as exogenous policies are compatible with an interior value $0<L_{c}(t)<1$. For the sake of illustration, when $\dot{\bar{u}}(t)=0$ as when no policies are implemented at all, the latter expression reduces to

$$
L_{c}(t)=\frac{\gamma \eta_{d}-\rho}{\gamma\left(\eta_{c}+\eta_{d}\right)}, \forall t \geq T
$$


which is lower than one as well as positive by the assumption that $\gamma \eta_{d}>\rho$.

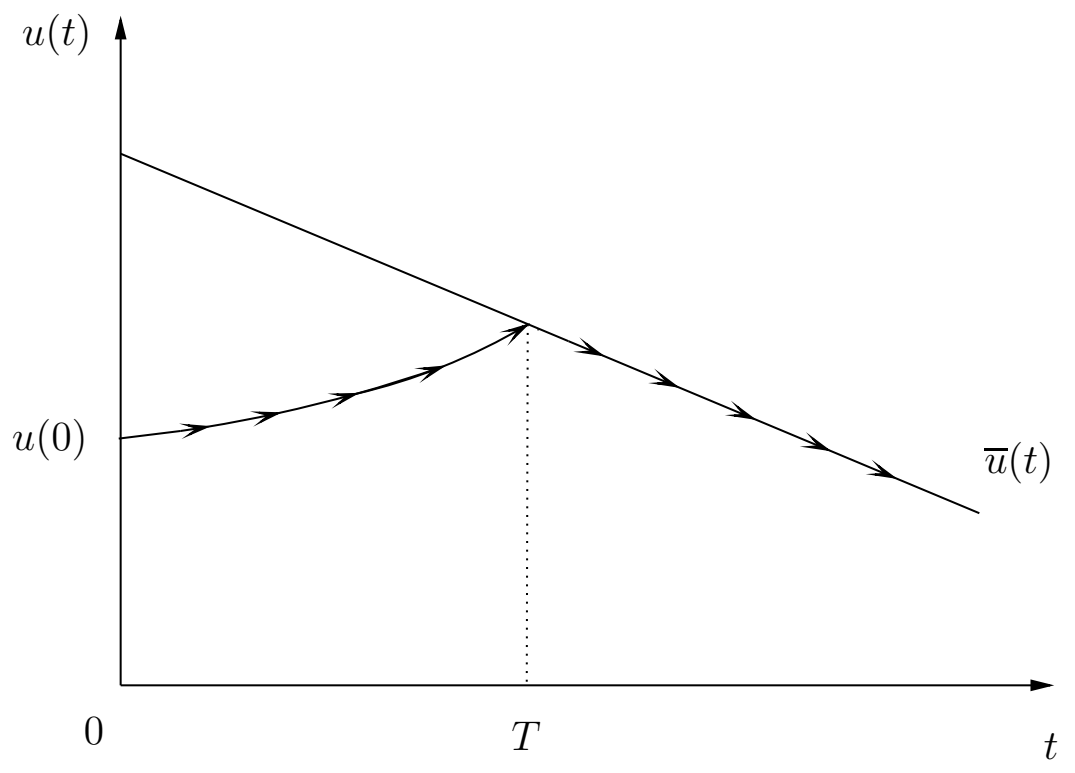

Figure 1: Convergence dynamics when $\varepsilon<1$

\section{Final remarks on the interior equilibrium: stability and symmetry}

It must be noted that the above interior equilibrium may not satisfy the desirable property of stability in the case where $\varepsilon>1$. This arises as a consequence of the simplifying and standard assumption that there are constant returns to scale in the labor input of $R \& D$ sectors.

A slightly more complex but otherwise analogous model would rule out the possibility that markets diverge from the interior equilibrium. Specifically, in presence of decreasing returns to scale in the labor input to $\mathrm{R} \& \mathrm{D}$ with the appropriate Inada type restrictions, in the spirit of Acemoglu (1998), corner allocations of scientists would be incompatible with noarbitrage market equilibrium; the above interior equilibrium would intuitively be the unique, stable equilibrium.

Finally, in any interior equilibrium, the within-R\&D-sector symmetry of scientists' allocation can be shown to arise endogenously. Indeed, the property shown in Appendix A that ratios $A_{j i}(t) / x_{j i}(t)$ are independent of $i \in[0,1]$ for each $j=c, d$, combined with the result (obtained in interior equilibrium) of Section 4 that $x_{j i}(t)=x_{j}(t)$, for all $i \in[0,1]$ and each $j=c, d$, implies that all $A_{c i}(t)$ and all $A_{d i}(t)$, for any $i \in[0,1]$, must always follow the same average trajectory. In light of (5), this also implies that scientists in each R\&D sector $j=c, d$ are evenly allocated over the range $i \in[0,1]: L_{j i}(t)=L_{j}(t)$. 


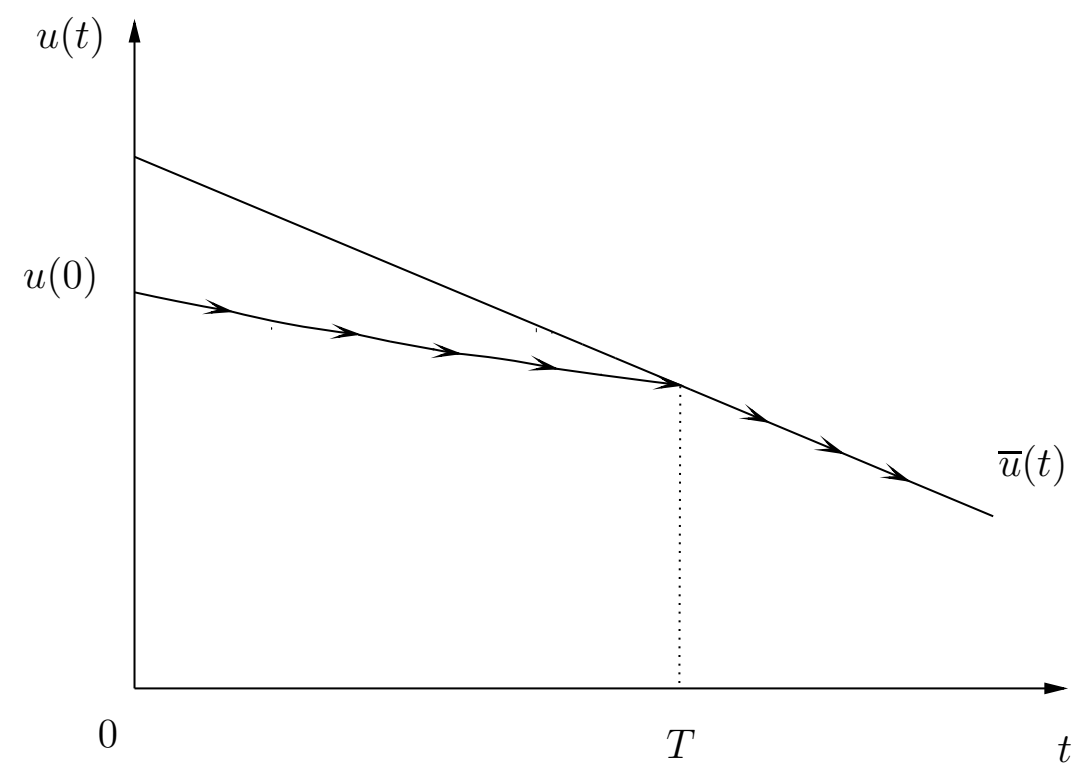

Figure 2: Convergence dynamics when $\varepsilon>1$

\section{Proof of (25)}

The proof given in the main text relies on the equality $g_{Y}(t)=g_{C}(t)$, which can be obtained as follows.

Substituting the price $p_{c i}(t)=\psi / \alpha$ from (15) into (12), and using the property shown in Appendix A that the ratios $A_{c i}(t) / x_{c i}(t)$ are independent of $i$ for all $i \in[0,1]$, so that they can be replaced by the ratio $A_{c}(t) / x_{c}(t)$, yield the relation $\left(\psi / \alpha^{2}\right) Y(t)^{-1 / \varepsilon}=$ $Y_{c}(t)^{-1 / \varepsilon}\left(Q A_{c}(t) / x_{c}(t)\right)^{1-\alpha}$.

Moreover, proceeding the same way as Appendix A with the same property that ratios $A_{j i}(t) / x_{j i}(t)$ are independent of $i \in[0,1]$ for each $j=c, d,(3)$ and (4) can respectively be expressed as follows:

$$
\begin{aligned}
Y_{c}(t) & =Q^{1-\alpha} A_{c}(t)^{1-\alpha} x_{c}(t)^{\alpha}, \\
Y_{d}(t) & =R(t)^{1-\alpha} A_{d}(t)^{1-\alpha} x_{d}(t)^{\alpha} .
\end{aligned}
$$

Using the simplified expression of sector $c$ 's production function (C.1), $\left(Q A_{c}(t) / x_{c}(t)\right)^{1-\alpha}$ can be replaced by $Y_{c}(t) / x_{c}(t)$. Replacing and rearranging, one immediately obtains the following expression:

$$
Y_{c}(t)^{(\varepsilon-1) / \varepsilon}=\frac{\psi}{\alpha^{2}} \frac{x_{c}(t)}{Y(t)^{1 / \varepsilon}} .
$$

Proceeding in the exact same way with variables relative to sector $d$ instead of $c$, by using (13) and (C.2), instead of (12) and (C.1), one obtains the symmetric formula

$$
Y_{d}(t)^{(\varepsilon-1) / \varepsilon}=\frac{\psi}{\alpha^{2}} \frac{x_{d}(t)}{Y(t)^{1 / \varepsilon}} .
$$


Introducing the expressions of $Y_{c}(t)^{(\varepsilon-1) / \varepsilon}$ and $Y_{d}(t)^{(\varepsilon-1) / \varepsilon}$ given by (C.3) and (C.4) into the production function (1), isolating the factor $\left(\left(\psi / \alpha^{2}\right) Y(t)^{-1 / \varepsilon}\right)^{\varepsilon /(\varepsilon-1)}$ and rearranging, all powers involving $\varepsilon$ cancel out to yield the expression

$$
Y(t)=\frac{\psi}{\alpha^{2}}\left(x_{c}(t)+x_{d}(t)\right)
$$

which we will use shortly below.

Finally, taking Equation (9), substituting $y_{j i}(t)=\psi x_{j i}(t)$ from (6), and making use of the notations $x_{c}(t)=x_{c i}(t)$ and $x_{d}(t)=x_{d i}(t)$, for all $i \in[0,1]$ (see in Section 4 the text immediately following Equation (20)), one obtains the following relation:

$$
C(t)=Y(t)-\psi\left(x_{c}(t)+x_{d}(t)\right) .
$$

By substitution of $\psi\left(x_{c}(t)+x_{d}(t)\right)$ from (C.5), we obtain the linear relation between $C(t)$ and $Y(t)$

$$
C(t)=Y(t)\left(1-\alpha^{2}\right)
$$

which implies that the growth rates of the two variables are identical in any equilibrium. 


\section{References}

Acemoglu, D. (1998), "Why Do New Technologies Complement Skills? Directed Technical Change and Wage Inequality", Quarterly Journal of Economics, 113: 1055-1089.

Acemoglu, D. (2002), "Directed Technical Change", Review of Economic Studies, 69: 781809.

Acemoglu, D. (2008), Introduction to Modern Economic Growth, Princeton University Press, Princeton.

Acemoglu, D., P. Aghion, L. Bursztyn and D. Hemous (2012), "The Environment and Directed Technical Change", American Economic Review, 102: 131-166.

André, F.J., and S.A. Smulders (2004), "Energy Use, Endogenous Technical Change and Economic Growth", mimeo.

Aghion, P., and P.W. Howitt (1992), "A Model of Growth through Creative Destruction", Econometrica, 60: 323-351.

Aghion, P., A. Dechezlepretre, D. Hemous, R. Martin and J. Van Reenen (2011) "Carbon Taxes, Path Dependency, and Directed Technical Change: Evidence from the Auto Industry", NBER Working Papers 18596.

Barro, R.J., and X. Sala-i-Martin (1995), Economic Growth, MIT Press, Cambridge.

Cozzi, G., P.E. Giordani and L. Zamparelli (2007), "The Refoundation of the Symmetric Equilibrium in Schumpeterian Growth Models", Journal of Economic Theory, 136: 788-797.

Dasgupta, P.S., G.M. Heal and J.E. Stiglitz (1981), "The Taxation of Exhaustible Resources", NBER Working Papers 436.

Daubanes, J., and P. Lasserre (2012), "Non-Renewable Resource Supply: Substitution Effect, Compensation Effect, and All That", CIRANO Working Papers 2012s-28.

Di Maria, C., and S. Valente (2008), "Hicks Meets Hotelling: The Direction of Technical Change in Capital-Resource Economies", Environment and Development Economics, 13: 691-717.

Gans, J.S. (2012), "Innovation and Climate Change Policy", American Economic Journal: Economic Policy, 4: 125-145.

Gaudet, G., and P. Lasserre (1990), "Dynamiques Comparées des Effets de la Taxation Minière", L'Actualité Economique, 66: 467-497.

Gerlagh, R. (2011), "Too Much Oil", CESifo Economic Studies, 57: 79-102. 
Grafton, R.Q., T. Kompas and N.V. Long (2012), "Substitution between Biofuels and Fossil Fuels: Is There a Green Paradox?", Journal of Environmental Economics and Management, 64: 328-341.

Grimaud, A., G. Lafforgue and B. Magné (2011), "Climate Change Mitigation Options and Directed Technical Change: A Decentralized Equilibrium Analysis", Resource and Energy Economics, 33: 938-962.

Grimaud, A., and L. Rougé (2008), "Environment, Directed Technical Change and Economic Policy", Environmental and Resource Economics, 41: 439-463.

Hart, R. (2004), "Growth, Environment and Innovation - A Model with Production Vintages and Environmentally Oriented Research", Journal of Environmental Economics and Management, 48: 1078-1098.

Hoel, M. (2010), "Is There a Green Paradox?", CESifo Working Papers 3168.

Pittel, K., and L. Bretschger (2010), "The Implications of Heterogeneous Resource Intensities on Technical Change and Growth", Canadian Journal of Economics, 43: 1173-1197.

Sinn, H.-W. (2008), "Public Policies Against Global Warming: A Supply Side Approach", International Tax and Public Finance, 15: 360-394.

Smulders, S.A., and C. Di Maria (2012), "The Cost of Environmental Policy under Induced Technical Change", CESifo Working Papers 3886.

van der Ploeg, F., and C.A. Withagen (2012), "Is There Really a Green Paradox?", Journal of Environmental Economics and Management, 64: 342-363.

Withagen, C.A. (1994), "Pollution and Exhaustibility of Fossil Fuels", Resource and Energy Economics, 16: 235-242. 